\title{
Some generalized Coxeter groups and their orbifolds
}

Marcel Hagelberg and Rubén A. Hidalgo

Abstract. In this note we construct examples of geometric 3-orbifolds

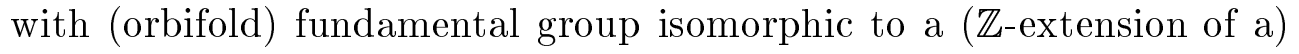
generalized Coxeter group. Some of these orbifolds have either euclidean, spherical or hyperbolic structure. As an application, we obtain an alternative proof of theorem 1 of Hagelberg, Maclaughlan and Rosenberg in [5]. We also obtain a similar result for generalized Coxeter groups.

\section{Introduction.}

A group with presentation

$$
\left\langle a_{1}, \ldots, a_{n}: a_{i}^{u_{i}}=\left(a_{i+1} a_{i}^{-1}\right)^{v_{i}}\right\rangle,
$$

where $u_{i}, v_{i}$ are integers greater or equal to two, is called a generalized Coxeter group. In the particular case $u_{i}=v_{i}=2$, the above is a Coxeter group with Coxeter diagram (see $[1$, p. 110]) as shown in figure 1.

A group having presentation

$$
\Gamma=\left\langle x_{1}, \ldots, x_{n}, t: x_{i}^{k_{i}}=\left(x_{i+1} x_{i}^{-1}\right)^{l_{i}}=\left(x_{i} t\right)^{m_{i}}=1\right\rangle,
$$

where $k_{i}, l_{i}, m_{i}$ are integers greater or equal to 2 , is called a $\mathbb{Z}$-extension of a generalized Coxeter group. The reason for the name is the following: 
if $H$ denotes the smallest normal subgroup of $\Gamma$ containing the generator $t$, then the quotient group $J=\Gamma / H$ has presentation

$$
J=\left\langle\tilde{x}_{1}, \ldots, \tilde{x}_{n}: \tilde{x}_{i}^{\widehat{k_{i}}}=\left(\tilde{x}_{i+1} \tilde{x}_{i}^{-1}\right)^{l_{i}}=1\right\rangle,
$$

where $\widehat{k_{i}}=\operatorname{mcd}\left(k_{i}, m_{i}\right)$, that is, $J$ turns out to be a generalized Coxeter group.

\section{Figure 1.}

Triangle and generalized tetrahedron groups are some examples of generalized Coxeter groups.

The main problem of three-orbifolds is their classification. As observed by W. Thurston in his project, geometry and topology are very well related, there are exactly eight geometries and essentially the conjecture is that all 3-orbifolds can be obtained by gluing a finite number of geometric orbifolds (that is, orbifolds of the form $X / G$, where $X$ is one of the eight geometries and $G$ is a discrete group of isometries of $X)$. The main geometry is given by the hyperbolic one.

To understand this classification problem is good to have examples of geometric orbifolds, which are the parts to be glued to form the more general ones. For it, triangle and Coxeter groups have been of great interest (see, for instance, in Coxeter-Moser [2]).

Another (hyperbolic) orbifolds, with generalized triangle groups as (orbifold) fundamental groups, were studied in [7], [4], [5] and [6], where results concerning their discreteness and arithmeticity were obtained.

Generalized Coxeter groups were studied from the group theorical perspective in [12] and [13]. 
The main idea of this paper is roughly speaking the following. We first describe some graphs in the 3-sphere (perhaps with some deleted vertices) together a data given by some integers. Secondly, we try to figure out which kind of geometric orbifolds can be obtained with these objects. For it, we construct certain planes intersecting in a very special fashion and we consider the reflections on these planes. We apply Poincare's polygon theorem to obtain that the group generated by those reflection is discrete and has the desired presentation (e.g. generators and relations).

More technically, we construct explicit embeddings of some $(\mathbb{Z}$ extension of) generalized Coxeter groups as group of isometries of some 3-dimensional geometry. The orbifolds obtained are then geometric 3-

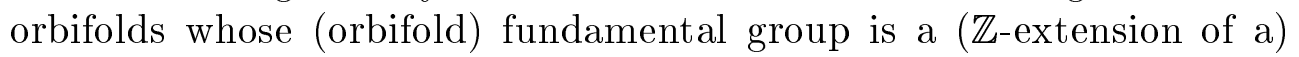
generalized Coxeter group.

The presented geometries in these constructions are $S^{2} \times \mathbb{R}, \mathbb{R}^{3}$, $\mathbb{H}^{2} \times \mathbb{R}$ and $\mathbb{H}^{3}$, where $S^{2}, \mathbb{H}^{2}$ and $\mathbb{H}^{3}$ denote the 2-sphere, the hyperbolic plane and the hyperbolic three space, respectively. Some of these orbifolds were obtained in the paper of Dunbar [3].

This paper is organized as follows. We start in Section 2 with some basics definitions and the description of a particular type of 3-orbifolds denoted by $\mathcal{O}(n, K, L, M)$. The essential property of these orbifolds is the fact that their (orbifold) fundamental groups are $\mathbb{Z}$-extension of generalized Coxeter groups.

In Section 3 we produce geometries of type $\mathcal{Z} \times \mathbb{R}$, for the orbifolds $\mathcal{O}(n, K, L, K)$ with $K=(2, \ldots, 2)$, where $\mathcal{Z} \in\left\{\mathbb{H}^{2}, \mathbb{R}^{2}, S^{2}\right\}$. More precisely, we obtain the following

Theorem 1. For $n \geq 3, K=(2, \ldots, 2)$ and $L=\left(l_{1}, \ldots, l_{n}\right)$, let $\kappa=n-2-\sum_{i=1}^{n} 1 / l_{i}$. Then the orbifold $\mathcal{O}(n, K, L, K)$ has geometry:
a) $\mathbb{H}^{2} \times \mathbb{R}$, if $\kappa>0$,
b) $S^{2} \times \mathbb{R}$, if $\kappa<0$,
c) $\mathbb{R}^{3}$, if $\kappa=0$.

We also obtain some generalized triangle groups, yielding part i) of Theorem 1 in [5]. More precisely, we prove that the group $\langle x, r$ : $\left.x^{2}=r^{n}=\left(r^{-1} x r x\right)^{l}=1\right\rangle$ can be embedded as group of isometries of $\mathbb{H}^{2} \times \mathbb{R}, S^{2} \times \mathbb{R}$ and $\mathbb{R}^{3}$ (respectively) if $(l-1) n-2 l$ is bigger, less and equal to zero (respectively). 
In Section 4 we construct generalized Coxeter groups, represented as $\mathbb{Z}_{2}$-extensions of Fuchsian groups of the first kind, that uniformize orbifolds $\mathcal{O}(n, K, L, K)$ with $K=(2, \ldots, 2), n \geq 3$ and $n-2>$ $\sum_{i=1}^{n} 1 / l_{i}$. We also construct the generalized triangle group $\left\langle x, r: x^{2}=\right.$ $\left.r^{n}=\left(r^{-1} x r x\right)^{l}=1\right\rangle$ as a $\mathbb{Z}$-extension of a Fuchsian group of the first kind for $(l-1) n-2 l>0$.

In Section 5 we generalize the construction of Section 4 to obtain $\mathbb{Z}_{2}$-extensions of Fuchsian groups (maybe of the second kind).

In Section 6 we produce hyperbolic structures for the orbifolds $\mathcal{O}(n, K, L, K)$, where $n \geq 5, K=(3, \ldots, 3)$ and $L=(2, \ldots, 2)$; more precisely, we prove the following.

Theorem 2. If $n \geq 5, K=(3, \ldots, 3)$ and $L=(2, \ldots, 2)$, then the orbifold $\mathcal{O}(n, K, L, K)$ has a hyperbolic structure.

We also show that for $n \geq 5$ the generalized triangle group $\langle x, r$ : $\left.x^{3}=r^{n}=\left(r^{-1} x^{-1} r x\right)^{2}=1\right\rangle$, can be realized as a group of hyperbolic isometries of $\mathbb{H}^{3}$.

In Section 7 we discuss the excluded cases $n \in\{3,4\}$ of Theorem 2.

In Section 8 we generalize the constructions of the sections 3-6. In this way, we can obtain a many generalized Coxeter groups as group of isometries of the hyperbolic three space. In some of these cases, one may proceed as in Section 6 to produce $\mathbb{Z}$-extensions of generalized Coxeter groups as groups of isometries of $\mathbb{H}^{3}$.

In Section 9 we use the construction done in Section 8 to obtain a simple alternative proof of [5, Theorem 1]. We obtain a similar result for generalized Coxeter groups. Namely, we have the following.

Theorem 3. Let $n, k, l$ be integers with $n \geq 2, l \geq 2$ and $k \geq 2$ and $(n, k, l) \neq(3,3,2)$. There is a discrete and faithful representation of the generalized Coxeter group

$$
G=\left\langle x_{1}, \ldots, x_{n}: x_{i}^{k}=\left(x_{i+1} x i^{-1}\right)^{l}=1\right\rangle,
$$

as group of isometries in one of the geometries $\mathbb{R}^{3}, \mathbb{H}^{2} \times \mathbb{R}, S^{2} \times \mathbb{R}$, $\mathbb{H}^{3}$. The geometry is $\mathbb{H}^{3}$ except when

$$
(n, k, l) \in\{(3,2,2),(3,2,3),(3,3,2),(4,2,2)\} .
$$


Part of this paper was written while the second author was visiting the Université Paul Sabatier.

\section{Some basic definitions.}

A $n$-orbifold $\mathcal{O}$ consists of a Hausdorff space $X$ (the underlying topological space of the orbifold) and a collection

$$
\left\{\left(U_{\alpha}, V_{\alpha}, G_{\alpha}, f_{\alpha}: U_{\alpha} \longrightarrow V_{\alpha} / G_{\alpha}\right), \alpha \in \mathcal{A}\right\},
$$

satisfying the following properties:

1) The collection $\left\{U_{\alpha}: \alpha \in \mathcal{A}\right\}$ is an open covering of $X$.

2) $G_{\alpha}$ is a finite group of homeomorphisms of the open subset $V_{\alpha} \subset \mathbb{R}^{n}$.

3) The map $f_{\alpha}: U_{\alpha} \longrightarrow V_{\alpha} / G_{\alpha}$ is a homeomorphism.

4) If $U_{\alpha} \cap U_{\beta} \neq \varnothing$, and if $\pi_{s}: V_{s} \longrightarrow V_{s} / G_{s}$ is the natural (branched) covering induced by the action of $G_{s}$ on $V_{s}$, then the map

$$
f_{\beta} \circ f_{\alpha}^{-1}: f_{\alpha}\left(U_{\alpha} \cap U_{\beta}\right) \longrightarrow f_{\beta}\left(U_{\alpha} \cap U_{\beta}\right)
$$

can be lifted to a homeomorphism

$$
h_{\alpha, \beta}: \pi_{\alpha}^{-1}\left(f_{\alpha}\left(U_{\alpha} \cap U_{\beta}\right)\right) \longrightarrow \pi_{\beta}^{-1}\left(f_{\beta}\left(U_{\alpha} \cap U_{\beta}\right)\right) .
$$

Figure 2. 
Some $\mathbb{Z}$-extensions of generalized Coxeter groups appear as (applying the Wirtinger algorithm and Haeflieger and Quach [8]) orbifold fundamental groups for orbifolds $\mathcal{O}(n, K, L, M)$ having the three sphere $S^{3}$ as underlying topological space and the planar graph $\pi_{n}(K, L, M)$ with the branching indices as shown in figure 2 as branching locus, where $K=\left(k_{1}, \ldots, k_{n}\right), L=\left(l_{1}, \ldots, l_{n}\right)$ and $M=\left(m_{1}, \ldots, m_{n}\right)$ are $n$-tuples of integers greater or equal to two. It may happen that one or both vertices of the graph are not there, in which case the underlying topological space is either $\mathbb{R}^{3}$ or $\mathbb{R}^{3}$ minus a point. Generators $X_{i}$ and $T$ for the (orbifold) homotopy group of $\mathcal{O}(n, K, L, M)$ are given by (homotopy classes of) the loops shown in figure 3.

Figure 3.

We remark that there are some necessary restrictions on the $n$ tuples $K, L, M$ in order to have an orbifold with the vertices included as described above. These restrictions are

$$
\left\{k_{i-1}, l_{i}, k_{i}\right\},\left\{m_{i-1}, l_{i}, m_{i}\right\} \in\{\{2,2, r\},\{2,3,3\},\{2,3,4\},\{2,3,5\}\},
$$

where $r \geq 2$. The above is consequence of the fact that the stabilizer of a point (to belong to the region of discontinuity of a discrete group) is a finite group which is either cyclic, dihedral or the group of isometries of a platonic solid.

A geometry is a simply-connected complete Riemannian manifold $(M, \rho)$ with transitive isometry group $G$, having a co-finite volume subgroup and such that the stabilizer at a point is compact. 
In dimension three, Thurston has shown that there are exactly eight geometries: $\mathbb{H}^{3}, S^{3}, \mathbb{R}^{3}, S^{2} \times \mathbb{R}, \mathbb{H}^{2} \times \mathbb{R}, \operatorname{sl}(2, \mathbb{R})$, Nil-geometry and Sol-geometry ([10], [1] and [11]).

\section{First construction: splitting geometries.}

We construct geometric structures for some of the above orbifolds $\mathcal{O}(n, K, L, K)$, where $K=(2,2, \ldots, 2)$ and $n \geq 3$. The geometries are given by $\mathbb{R}^{3}, S^{2} \times \mathbb{R}$ and $\mathbb{H} \times \mathbb{R}$. In this way, the corresponding $\mathbb{Z}$-extension generalized Coxeter groups can be realized as a group of isometries in the respective geometry. The case $n=2$ (where necessarily, $l_{1}=l_{2}$ ) and some cases for $n=3$ were considered by Dunbar in [3]. The above is summarized in the following.

Theorem 1. For $n \geq 3, K=(2, \ldots, 2)$ and $L=\left(l_{1}, \ldots, l_{n}\right)$, let $\kappa=n-2-\sum_{i=1}^{n} 1 / l_{i}$. Then the orbifold $\mathcal{O}(n, K, L, K)$ has geometry:
a) $\mathbb{H}^{2} \times \mathbb{R}$, if $\kappa>0$,
b) $S^{2} \times \mathbb{R}$, if $\kappa<0$,
c) $\mathbb{R}^{3}$, if $\kappa=0$.

Proof. Set $\mathcal{Z}$ equal to $S^{2}, \mathbb{R}^{2}$ and $\mathbb{H}^{2}$ for $\kappa$ negative, positive and zero, respectively. We consider a geodesic polygon $P \subset \mathcal{Z}$ of $n$-sides and internal angles equals to (in cyclic order) $\pi / l_{1}, \ldots, \pi / l_{n}$, respectively. We label the sides of $P$ as $e_{1}, \ldots, e_{n}$, in such a way that $e_{i}$ connects the vertices with angles $\pi / l_{i-1}$ and $\pi / l_{i}$. Let $\sigma_{i}$ be reflection on the geodesic line containing the side $e_{i}$. We define the following isometries of $X \times \mathbb{R}$

$$
x_{i}(p, q)=\left(\sigma_{i}(p), q\right) \quad \text { and } \quad t(p, q)=(p, q+1),
$$

where $(p, q) \in \mathcal{Z} \times \mathbb{R}$.

The group $\Gamma$ generated by $x_{1}, \ldots, x_{n}$ and $t$ is a discrete group acting on $\mathcal{Z} \times \mathbb{R}$ with fundamental domain given by $P \times[-1 / 2,1 / 2]$ (the transformations $x_{1}, \ldots, x_{n}$ generate a discrete group acting on $X \times\{q\}$ with fundamental polygon $P \times\{q\}$ for all $q \in \mathbb{R}$, and $t$ is just an orthogonal translation to $X$. In fact, this is just Poincare's Polyhedron theorem on $X \times \mathbb{R})$.

It is evident that the above group is in fact a generalized Coxeter group and that the quotient $(\mathcal{Z} \times \mathbb{R}) / \Gamma$ is an orbifold with $S^{3}$ as 
underlying space and as branching set the planar graph $\pi_{n}(K, L, M)$, where $K=M$ is the $n$-tuple formed by 2 at each component and $L=\left(l_{1}, \ldots, l_{n}\right)$.

REMARK 1. Suppose that in the above construction we have the particular situation $l_{i}=l$, for some fixed $l \geq 2$. In this case, we may consider the polygon $P$ to be invariant under an isometry $\tilde{r}$ of $\mathcal{Z}$ of order $n$. Set $r(p, q)=(\tilde{r}(p), q)$. We have that $r$ is an isometry of $X \times \mathbb{R}$ of order $n$, keeping invariant $P \times[-1 / 2,1 / 2]$. If we set $x=x_{1}$, then the group $\tilde{\Gamma}$ generated by $\Gamma$ and $r$ has the following presentation

$$
\tilde{\Gamma}=\left\langle x, t, r: x^{2}=r^{n}=(r t)^{2}=\left(r^{-1} x r x\right)^{l}=r t r^{-1} t^{-1}=I\right\rangle .
$$

The geometric orbifold uniformized by $\tilde{\Gamma}$ is shown in figure 4a. The group $G$ generated by $x$ and $r$ has presentation

$$
G=\left\langle x, r ; x^{2}=r^{n}=\left(r^{-1} x r x\right)^{l}=1\right\rangle,
$$

(a generalized triangle group) and a fundamental domain given by the infinite volume cylinder $P^{*} \times \mathbb{R}$, where $P^{*}$ is the triangle bounded $e_{1}$ and the two (geodesic) lines connecting the fixed point of $\tilde{r}$ with both ends of $e_{1}$. It follows that the geometric orbifold uniformized by $G$ has infinite covolume, underlying topological space $S^{3}$ minus two points and branching locus has shown in figure 4 b. In particular, if $\mathcal{Z}$ is either $S^{2}$ or $\mathbb{R}^{2}$, then we obtain (with the exception of one case) the cases in $[5$, Theorem 1.i)]. 


\section{Second construction: uniformizations by $\mathbb{Z}_{2}$-extensions of Fuchsian groups.}

If in Theorem 1 the geometry is $\mathbb{H}^{2} \times \mathbb{R}$ (that is, if $n \geq 3$ and $\left.n-2-\sum_{i}^{n} 1 / l_{i}>0\right)$ we can uniformize the orbifolds $\mathcal{O}(n, K, L, K)$, where $K=(2, \ldots, 2)$ and $L=\left(l_{1}, \ldots, l_{n}\right)$, by the manifold $M=S^{3}-\widehat{\mathbb{R}}$, where $\widehat{\mathbb{R}}=\mathbb{R} \cup\{\infty\}$. We can construct $J$ so that $\mathcal{O}(n, K, L, K)=M / J$ and $J$ is a group of Möbius transformations, in fact a $\mathbb{Z}_{2}$-extension of a Fuchsian group. The group $J$ is constructed as follows: in the hyperbolic three space $\mathbb{H}^{3}=\{(z, t) \in \mathbb{C} \times \mathbb{R}, t>0\}$ we consider the model of hyperbolic two space $\mathbb{H}_{*}^{2}=\left\{(z, t) \in \mathbb{H}^{3}, \operatorname{Im}(z)=0\right\}$.

On $\mathbb{H}_{*}^{2}$ we consider a geodesic polygon $P$ of $n$ sides and consecutive angles $\pi / l_{1}, \ldots, \pi / l_{n}$.

Let $e_{i}$ be the side of $P$ determined by the vertices with angles $\pi / l_{i-1}$ and $\pi / l_{i}$.

Set $x_{i}$ the isometry of $\mathbb{H}^{3}$ of order two having fixed point axes containing $e_{i}$.

Set $D$ to be the common region determined by the geodesic planes $F_{1}, \ldots, F_{n}$, orthogonal to $\mathbb{H}_{*}^{2}$, such that $F_{i} \cap \mathbb{H}_{*}^{2}$ contains $e_{i}$.

As a consequence of Poincare's Polyhedron theorem [9], the group $J$, generated by $x_{1}, \ldots, x_{n}$, is a discrete group with $D$ as a fundamental polyhedron.

The group $J$ also acts as group of isometries of $\mathbb{H}_{*}^{2}$ (the transformations $x_{i}$ are reflections) with $P$ as compact fundamental domain for its action. It follows that the limit set of $J$ is the boundary $\gamma$ of $\mathbb{H}_{*}^{2}$ and, in particular, the group $J$ is either a Fuchsian group or a $\mathbb{Z}_{2^{-}}$extension of a Fuchsian group of the first kind. Since each generator $x_{i}$ permutes both discs on $\widehat{\mathbb{C}}$ (the boundary of $\mathbb{H}^{3}$ ) bounded by $\gamma$, we have that $J$ is a $\mathbb{Z}_{2^{-}}$extension of a Fuchsian group of the first kind.

It is not hard to see that $J$ is a generalized Coxeter group (with the generators $\left.x_{1}, \ldots, x_{n}\right)$ and that $\left(S^{3}-\widehat{\mathbb{R}}\right) / J$ is the orbifold $\mathcal{O}(n, K, L, K)$.

REMARK 2. As before, by considering $l_{i}=l>n /(n-2)$, we may assume the geodesic polygon to be invariant under a rotation $\tilde{R}$ of order $n$. By performing the same construction as in Remark 1, we obtain an uniformization of the orbifold shown in figure 4 a by a group $G$ with presentation $\left(x=x_{1}\right)$

$$
G=\left\langle x, r: x^{2}=r^{n}=\left(x r x r^{-1}\right)^{l}=I\right\rangle,
$$

that is, a generalized triangle group (compare to [5, Theorem 1]). 


\section{Generalization of the second construction.}

The above construction can be generalized as follows. Let $P$ be a hyperbolic polygon, maybe with an infinite number of sides, in the hyperbolic plane $\mathbb{H}_{*}^{2} \subset \mathbb{H}^{3}$.

We assume each internal angle $\theta_{i}=\pi / l_{i}$, where $l_{i}$ is an integer greater or equal to two.

For each side $e_{j}$ of $P$, we consider the isometry $x_{j}$ of order two, with fix point set the geodesic containing $e_{j}$. Set $G$ the group generated by all the transformations $x_{j}$.

A geodesic polyhedron $Q$ is defined by the common region bounded by the geodesic planes $F_{i}$, orthogonal to $\mathbb{H}_{*}^{2}$, such that $F_{i} \cap \mathbb{H}_{*}^{2}$ contains $e_{i}$.

Poincare's Polyhedron theorem [9] applied to $Q$ and the side pairings $x_{j}$ asserts that $G$ is a discrete group, $Q$ is a fundamental polyhedron for the action of $G$, and a minimal set of relations for $G$ given by $\left(x_{j-1} x_{j}\right)^{l_{j}}=I$ (if the sides $e_{j-1}$ and $e_{j}$ meet in $\mathbb{H}_{*}^{2}$ at the angle $\pi / l_{j}$ ).

The group $G$ is again a $\mathbb{Z}_{2}$-extension of a Fuchsian group, and is of the first kind if and only if the polygon $P$ has no sides in the boundary $\widehat{\mathbb{C}}$. In the case that $G$ is not of the first kind, the orbifold $\left(S^{3}-\Lambda\right) / G$, where $\Lambda$ is the limit set of $G$, has universal covering $S^{3}-\Lambda$. It has (orbifold) fundamental group isomorphic to $G$.

\section{Third construction: hyperbolic uniformization.}

One may think that the type of orbifolds we are considering cannot have a hyperbolic structure, that is, there is not a discrete group $\Gamma$ of isometries of $\mathbb{H}^{3}$ which is a $\mathbb{Z}$-extension of a generalized Coxeter group.

The following theorem asserts that this is not the case, that is, we may have hyperbolic structures on some of our orbifolds.

Theorem 2. If $n \geq 5, K=M=(3, \ldots, 3)$ and $L=(2, \ldots, 2)$, then the orbifold $\mathcal{O}(n, K, L, M)$ has a hyperbolic structure.

Proof. Set $n \geq 5, K=M=(3, \ldots, 3), L=(2, \ldots, 2)$ and choose as $\alpha$ the angle between two faces of the regular Euclidean tetrahedron (that is, $\alpha \in(0, \pi)$ with $\cos \alpha=1 / 3)$.

We continue to consider the hyperbolic plane in the three space $\mathbb{H}_{*}^{2}=\left\{(z, t) \in \mathbb{H}^{3}, \operatorname{Im}(z)=0\right\}$. 
Let $r$ be an isometry of $\mathbb{H}^{3}$ of order $n$, keeping invariant the plane $\mathbb{H}_{*}^{2}$.

We consider a hyperbolic polygon of $n$ sides $P \subset \mathbb{H}$ which is invariant under the action of $r$. We label the sides of $P$ consecutively in counterclockwise order by $e_{1}, \ldots, e_{n}$, respectively.

We may assume all internal angles to be equal to $\alpha$. This is consequence of the fact that $\alpha<\pi / 2$.

Choose a hyperbolic isometry $x_{1}$ of order three having as its fixed points set the geodesic line containing $e_{1}$. Since there are two possible choices for $x_{1}$, we take the one for which $x_{1}(P)$ consists of points $(z, t)$ with $\operatorname{Im}(z)>0$. Set $x_{i+1}=r x_{i} r^{-1}$.

The choice of the angle $\alpha$ ensures that both $x_{i}$ and $x_{i+1}$ generate the alternating group $A_{4}$. Moreover, every relation of $A_{4}$ is consequence of the relations $x_{i}^{3}=x_{i+1}^{3}=\left(x_{i+1} x_{i}^{-1}\right)^{2}=I$.

Consider the totally geodesic half-plane $F_{1}^{+}$containing the side $e_{1}$, making an angle of $\pi / 3$ with $\mathbb{H}_{*}^{2}$ and such that $F_{1}^{-}=x_{1}\left(F_{1}^{+}\right)$also makes an angle of $\pi / 3$ with $\mathbb{H}_{*}^{2}$ (see figure 5 ). The half-planes $F_{i}^{*}=r^{i-1}\left(F_{1}^{*}\right)$, for $* \in\{+,-\}$ and $i=1, \ldots, n$, determine a hyperbolic polyhedron $P^{3}$ with $2 n$ sides.

Figure 5 .

We have that $x_{i}$ is equal to $\sigma \circ \sigma_{i}$, where $\sigma_{i}$ is reflection on $F_{i}^{+}$and $\sigma$ is reflection on $\mathbb{H}_{*}^{2}$.

The transformations $x_{1}, \ldots, x_{n}$, pair the sides by the rule $x_{i}\left(F_{i}^{+}\right)=$ $F_{i}^{-}$. It is easy to check that the sides $F_{i}^{*}$ and $F_{i+1}^{*}$ meet at a right angle: it is a consequence of the fact that the transformation $x_{i+1} x_{i}^{-1}$ 
has order two.

Poincare's Polyhedron theorem [9] asserts that the group $G$ generated by these transformations is a Kleinian group with $P^{3}$ as fundamental domain.

Figure 6.

\section{Figure 7.}

The intersection of $P^{3}$ with the boundary $\widehat{\mathbb{C}}$ of $\mathbb{H}^{3}$ consists of two right hyperbolic polygons $P_{1}$ and $P_{2}$, each one of $n$ sides, such that each side of $P_{1}$ is paired by the transformations $x_{i}$ to one side of $P_{2}$ (see figure 6). Each of these two polygons belongs to some component of 
the region of discontinuity of $G$ which must be a round disc. The (circle) boundaries of these two components are disjoint, but both components are equivalent under $G$. It follows that $G$ has no invariant component, each component is a round disc and they are all equivalent under the action of $G$. We also have that $\left(\mathbb{H}^{3} \cup \Omega(G)\right) / G$ is topologically the unit three ball with the boundary and branching set the planar graph shown in figure 7 .

We may assume that the polygon $P_{1}$ is the one formed by the faces $F_{1}^{+}, \ldots, F_{n}^{+}$. The (circle) boundary of the component of the region of discontinuity of $G$ containing such a polygon determines a geodesic plane $W$ on $\mathbb{H}^{3}$. The plane $W$ necessarily cuts $P^{3}$ orthogonally. Let $j$ be the reflection on $W$ and set $t=j \sigma j \sigma$.

Set $Q$ to be the hyperbolic polyhedron determined by the sides of $P^{3}$ and the two geodesic planes $j\left(\mathbb{H}_{*}^{2}\right)$ and $\sigma\left(j\left(\mathbb{H}_{*}^{2}\right)\right)$, respectively. Side pairings of $Q$ are given by $x_{1}, \ldots, x_{n}$ and $t$.

The conditions for Poincare's polyhedron theorem [9] are satisfied in this case. In particular, we obtain that the group $\Gamma$ generated by these transformations is a discrete group, $Q$ is a fundamental polyhedron, and $G$ is a $\mathbb{Z}$-extension of a generalized Coxeter group. Moreover, the orbifold $\mathbb{H}^{3} / \Gamma$ is an orbifold with underlying topological space $S^{3}$ and branching set the planar graph $\pi_{n}(K, L, M)$ with $K, L, M$ as before.

\section{Figure 8.}

REMARK 3. The group $\tilde{\Gamma}$ generated by $\Gamma$ and the elliptic transformation $r$ is again a hyperbolic group with the presentation

$$
\tilde{\Gamma}=\left\langle x, t, r: x^{3}=r^{n}=(x t)^{3}=\left(r^{-1} x^{-1} r x\right)^{2}=t^{-1} r^{-1} t r=I\right\rangle,
$$


where $x=x_{1}$. It uniformizes the orbifold shown in figure 8a. The group $G$ generated by $x$ and $r$ has the presentation

$$
G=\left\langle x, r: x^{3}=r^{n}=\left(r^{-1} x^{-1} r x\right)^{2}=1\right\rangle,
$$

that is, a generalized triangle group. It uniformizes a hyperbolic orbifold with $S^{3}$ minus a point as underlying topological space, and branch locus as shown in figure $8 \mathrm{~b}$.

\section{The excluded cases $n=3$ and $n=4$ in theorem.}

\subsection{The case $n=4$.}

Let us consider the orbifold $\mathcal{O}(4, K, L, K)$, where $K=(3,3,3,3)$, $L=(2,2,2,2)$. For this, we proceed to construct in the hyperbolic plane $\mathbb{H}_{*}^{2} \subset \mathbb{H}^{3}$ a hyperbolic quadrilateral $P$, invariant under the rotation $r$ of order 4 with fixed points axis orthogonal to $\mathbb{H}_{*}^{2}$.

In the same way as in Section 6, we obtain a hyperbolic polyhedron $P^{3}$ with 8 sides, labeled $F_{i}^{+}$and $F_{i}^{-}$and side pairings $x_{i}$ for $i=1, \ldots, 4$. In this case, we have that the sides $F_{1}^{q}, F_{2}^{q}, F_{3}^{q}$ and $F_{4}^{q}$, with $q \in\{+,-\}$, meet at a point $p_{q} \in \widehat{\mathbb{C}} \cap A(r)$, where $A(r)$ is the set of fixed points of $r$.

We choose $W$ to be a horosphere centered at $p_{+}$and disjoint from $\mathbb{H}_{*}^{2}$, and denote by $j$ and $\sigma$ the reflections (anticonformal involutions of $S^{3}$ ) across $W$ and $\mathbb{H}_{*}^{2}$, respectively. 
Set $t=j \sigma j \sigma$, and consider $Q$ to be the region bounded by $P^{3}$ and the spheres $j\left(\mathbb{H}_{*}^{2}\right)$ and $\sigma\left(j\left(\mathbb{H}_{*}^{2}\right)\right)$. Denote by $\tilde{P}^{3}$ the image of the polyhedron $P^{3}$ under the reflection on the sphere $\widehat{\mathbb{C}}$.

The group $\Gamma$ generated by $x_{1}, \ldots, x_{4}$ and $t$ is a Kleinian group acting on $S^{3}$ for which the boundary sphere of $\mathbb{H}^{3}$ is contained in its limit set.

This group has exactly one invariant component $\Delta \subset \mathbb{H}^{3}$ which is simply connected.

We have $Q \subset \Delta$ is a fundamental domain for the action of $\Gamma$ on $\Delta$. The orbifold $\Delta / \Gamma$ is $\mathcal{O}(n, K, L, K)$. The other components are all equivalent to the complement of the closure of $\mathbb{H}^{3}$ in $S^{3}$, denoted by $\mathbb{H}_{-}^{3}$. The stabilizer of $\mathbb{H}_{-}^{3}$ is the group $G$ generated by $x_{1}, \ldots, x_{4}$. The orbifold $\mathbb{H}_{-}^{3} / G$ is shown in figure 9 .

\section{REMARK 4.}

1) The group $\Gamma$ is the analogous to a regular B-group in dimension 2 (a Kleinian group with a simply connected invariant component [9]).

2) The group $\tilde{\Gamma}$ generated by $r$ and $\Gamma$ uniformizes the orbifolds shown in figure 10.

Figure 10.

3) The group $G$ generated by $x=x_{1}$ and $r$ has presentation

$$
G=\left\langle x, r: x^{3}=r^{4}=\left(r^{-1} x^{-1} r x\right)^{2}=1\right\rangle,
$$

that is, $G$ is a generalized triangle group. It uniformizes the hyperbolic orbifold of figure $10 \mathrm{~b}$. 


\subsection{The Case $n=3$.}

Let us consider now the orbifold $\mathcal{O}(3, K, L, K)$, where $K=(3,3,3)$, $L=(2,2,2)$. In this case the orbifold fundamental group has the representation

$$
\Gamma=\left\langle x_{1}, x_{2}, x_{3}, t ; x_{i}^{3}=\left(x_{i+1} x_{i}^{-1}\right)^{2}=\left(x_{i} t\right)^{3}=1\right\rangle .
$$

In this case, the subgroup $G$ generated by the transformations $x_{1}, x_{2}$ and $x_{3}$ is a finite group of order 60 . Moreover, it has the representation

$$
G=\left\langle x_{1}, x_{2}, x_{3}: x_{i}^{3}=\left(x_{i+1} x_{i}\right)^{2}=1\right\rangle .
$$

It follows that $G$ has no elements of order 5 . In particular, we cannot have an embedding of the group $G$ (so neither of $\Gamma$ ) as group of isometries of $S^{3}, \mathbb{H}^{3}, \mathbb{R}^{3}, S^{2} \times \mathbb{R}, \mathbb{H}^{2} \times \mathbb{R}, \operatorname{sl}(2, \mathbb{R})$.

Theorem 1 (parts (2.a) and (3)) in [3] asserts that our orbifold cannot have neither Nil nor Sol geometry and, in particular, the group $\Gamma$ cannot be embedded as group of isometries of any of these two geometries.

\section{Generalization of the Constructions.}

In this section, we generalize the previous constructions. These generalizations give embeddings of generalized Coxeter groups as group of isometries of the hyperbolic three space. In many of the cases, they also produce embeddings of $\mathbb{Z}$-extensions of generalized Coxeter groups as isometries of $\mathbb{H}^{3}$.

We use the unit three ball in $\mathbb{R}^{3}$ as model of the hyperbolic three space, that is, $\mathbb{H}^{3}=\left\{(x, y, z) \in \mathbb{R}^{3}: x^{2}+y^{2}+z^{2}<1\right\}$. We need the following basic fact:

Lemma. Let $F_{1}, F_{2}$ and $F_{3}$ be three hyperbolic planes in $\mathbb{H}^{3}$. We assume that the three planes intersect in a point $q \in \mathbb{H}^{3} \cup \widehat{\mathbb{C}}$. Denote by $l_{i}$ the intersection between the planes $F_{3}$ and $F_{i}$, for $i=1,2$. For $q \in \widehat{\mathbb{C}}$, set $\alpha=0$, otherwise set $\alpha \in(0, \pi)$ to be the angle between $l_{1}$ and $l_{2}$ (we use counterclockwise orientation in the plane determined by those two lines). If $\beta_{i} \in(0, \pi / 2)$ is the angle between $F_{i}$ and $F_{3}$, and $\theta \in[\alpha, \pi]$ is the angle between $F_{1}$ and $F_{2}$, then

$$
\cos \theta=-\cos \beta_{1} \cos \beta_{2}+\cos \alpha \sin \beta_{1} \sin \beta_{2} .
$$


Figure 11 shows the situation described in the above lemma.

Figure 11.

Proof. If $q \in \widehat{\mathbb{C}}$, then we may assume $q=\infty$. In this case, the planes are orthogonal euclidean planes in $\mathbb{R}^{3}$ to the plane $\left\{(x, y, z) \in \mathbb{R}^{3}: z=\right.$ $0\}$, and the three planes $F_{1}, F_{2}$ and $F_{3}$ determine a euclidean triangle of internal angles $\beta_{1}, \beta_{2}$ and $\theta$ and, in particular, the above formula holds trivially.

Let us assume now that $q$ is inside the hyperbolic three space. In this case, we may assume the model of $\mathbb{H}^{3}$ given by the unit three ball in $\mathbb{R}^{3}$ with center in the origin $q=0$. We also may assume that $-x / y\}$,

a) $l_{1}=\{(x, y, z): x=z=0\}, l_{2}=\{(x, y, z): z=0, \tan \alpha=$

b) $F_{3}=\{(x, y, z): z=0\}$,

c) $F_{1}^{\perp}=\left\langle w_{1}\right\rangle$, where

$$
w_{1}=\left(\cos \left(\pi / 2-\beta_{1}\right), 0, \sin \left(\pi / 2-\beta_{1}\right)\right),
$$

d) $F_{2}^{\perp}=\left\langle w_{2}\right\rangle$, where

$$
w_{2}=\left(\cos \alpha \cos \left(\pi / 2-\beta_{2}\right),-\sin \alpha \cos \left(\pi / 2-\beta_{2}\right),-\sin \left(\pi / 2-\beta_{2}\right)\right) .
$$

Now the equality $w_{1} \cdot w_{2}=\cos \theta$ gives the desired equality.

Now we proceed to the construction. For this, let us consider two $n$-tuples $K=\left(k_{1}, \ldots, k_{n}\right)$ and $L=\left(l_{1}, \ldots, l_{n}\right)$ of integers bigger or 
equal to two, such that

$$
\frac{1}{l_{i}}+\frac{1}{k_{i}}+\frac{1}{k_{i+1}} \geq 1
$$

We determine angles $\alpha_{i} \in\left[0, \pi / l_{i}\right]$ by the equation

$$
\cos \alpha_{i}=\frac{\cos \left(\frac{\pi}{l_{i}}\right)+\cos \left(\frac{\pi}{k_{i}}\right) \cos \left(\frac{\pi}{k_{i+1}}\right)}{\sin \left(\frac{\pi}{k_{i}}\right) \sin \left(\frac{\pi}{k_{i+1}}\right)} .
$$

The last equation gives some restrictions on $l_{i}, k_{i}$ and $k_{i+1}$. We assume that

$$
(n-2) \pi>\sum_{i=1}^{n} \alpha_{i}
$$

On the hyperbolic plane $\mathbb{H}^{2}=\left\{(x, y, z) \in \mathbb{H}^{3}: z=0\right\}$, we draw an $n$-sided hyperbolic polygon $P$, where the sides are labeled cyclically as $e_{1}, e_{2}, \ldots, e_{n}$. We assume that the vertex $v_{i}$, determined by $e_{i}$ and $e_{i+1}$, is contained inside $\mathbb{H}^{3}$ or on its boundary according as

$$
\frac{1}{l_{i}}+\frac{1}{k_{i}}+\frac{1}{k_{i+1}}
$$

is bigger than one or equal to one. The (internal) angle of the vertex $v_{i}$ is $\alpha_{i}$.

The existence of such a polygon is guaranteed by the inequality (iii). For each edge $e_{i}$, we consider a hyperbolic plane $F_{i}$ that contains $e_{i}$. Let $F_{i}^{+}$be the part of the above plane contained in the half-space $\{z \geq 0\}$. We assume that the angle between $F_{i}^{+}$and $P$ is exactly $\pi / k_{i}$.

Set $\sigma$ and $\sigma_{i}$ to be the reflection through $\mathbb{H}^{2}$ and $F_{i}$, respectively. Let $K$ be the group generated by the reflections $\sigma, \sigma_{1}, \ldots, \sigma_{n}$. Since the angle between the planes $F_{i}^{+}$and $F_{i+1}^{+}$is $\pi / l_{i}$ (this is a consequence of the above lemma and the definition of $\alpha_{i}$ ), and the polyhedron $P^{3}$ determined by the faces $P, F_{1}^{+}, \ldots, F_{n}^{+}$, has finite number of sides, Poincare's polyhedron theorem applies to this case to obtain that:

a) $K$ is a discrete group of isometries.

b) $P^{3}$ is a fundamental domain for $K$. 
c) The generators for $K$ are $\sigma, \sigma_{1}, \ldots, \sigma_{n}$.

d) A set of maximal relations on these generators are: $\sigma^{2}=\sigma_{i}^{2}=$ $\left(\sigma \sigma_{i}\right)^{k_{i}}=\left(\sigma_{i+1} \sigma_{1}\right)^{l_{i}}=1$.

If we set $x_{i}=\sigma \sigma_{i}$, then we have that the group $J$ generated by $x_{1}, \ldots, x_{n}$ has the presentation

$$
J=\left\langle x_{1}, \ldots, x_{n}: x_{i}^{k_{i}}=\left(x_{i+1} x_{i}^{-1}\right)^{l_{i}}=1\right\rangle,
$$

that is, $J$ is a generalized Coxeter group. A fundamental polyhedron for $J$ is given by $Q=P^{3} \cup \sigma\left(P^{3}\right)$, and the index of $J$ in $K$ is two.

In the particular cases that $Q$ intersects the boundary of $\mathbb{H}^{3}$ in two polygons (both symmetric by the reflection $\sigma$ ), they will have internal angles equal to $\pi / l_{1}, \ldots, \pi / l_{n}$. If it happens that $(n-2) \pi>\sum_{i=1}^{n} \pi / l_{i}$, and the group $J$ is not Fuchsian group, then we may proceed as in construction of Section 6 to obtain a $\mathbb{Z}$-extension of a generalized Coxeter group.

\section{An application.}

In this section we proceed to obtain an alternative and easy proof of [5, Theorem 1], and an equivalent result for generalized Coxeter groups.

\subsection{First construction.}

We continue using the notation of Section 8, and assume $n \geq 3$, $l_{i}=l \geq 2$ and $k_{i}=k \geq 2$. In this situation the restrictions to the construction are the following:

i) $1 / l+2 / k \geq 1$.

ii) The equation

$$
\cos \alpha=\frac{\cos \left(\frac{\pi}{l}\right)+\cos ^{2}\left(\frac{\pi}{k}\right)}{\sin ^{2}\left(\frac{\pi}{k}\right)}
$$

has a solution in the interval $[0, \pi / l]$.

iii) $(n-2) \pi / n>\alpha$. 
In this case, the restriction ii) is a consequence of $i$ ), and restriction iii) is equivalent to:

iii.1) If $n=3$, then

$$
2 \cos \left(\frac{\pi}{l}\right)>1-3 \cos ^{2}\left(\frac{\pi}{k}\right) .
$$

iii.2) If $n=4$, then

$$
\sqrt{2} \cos \left(\frac{\pi}{l}\right)>1-(1+\sqrt{2}) \cos ^{2}\left(\frac{\pi}{k}\right) .
$$

iii.3) If $n \geq 5$, then there is no restriction.

In particular, the only exceptional cases for $(n, k, l)$ are those with $1 / l+2 / k<1$ and $(3,2,2),(3,3,2),(3,2,3)$ and $(4,2,2)$.

We may construct the polygon $P$ (as done in Section 8) with $n$ sides, all internal angles equal to $\alpha$, and invariant under an isometry $R$ of order $n$. The group $G$ generated by $x=x_{1}$ (constructed as in last section) and $r$ is a group of isometries of $\mathbb{H}^{3}$ with presentation

$$
G=\left\langle x, r: x^{k}=r^{n}=\left(r^{-1} x^{-1} r x\right)^{l}=1\right\rangle .
$$

Set $M^{+}$and $M^{-}$the geodesic planes determined by the following properties:

1) $M^{+}$and $M^{-}$are orthogonal to $\mathbb{H}^{2}$.

2) $M^{+} \cap \mathbb{H}^{2}$ and $M^{-} \cap \mathbb{H}^{2}$ are geodesics through the vertices of $P$ determined by the side $e_{1}$.

3) The angle between $M^{+}$and $M^{-}$is $2 \pi / n$. 
The hyperbolic polygon determined by the faces $F_{1}^{+}, F_{1}^{-}$(as in last section) and the two hyperbolic planes $M^{+}, M^{-}$is a fundamental polyhedron for $G$. The orbifold uniformized by $G$ is shown in figure 12, where one or the two vertices may be deleted. The only cases for which the above orbifolds have finite volume are given when $n \in\{3,4\}$ (these two are the only cases when such a polyhedron does not meet the boundary with non-empty interior).

\subsection{Second construction.}

Now we consider the case $(n, k, l)$ such that

$$
\frac{1}{l}+\frac{2}{k}<1
$$

Let us consider the restrictions

i) If $n=3$, then $l \geq 4$.

ii) If $n=4$, then $l \geq 3$.

iii) If $n \geq 5$, then $l \geq 2$.

Draw in the complex plane a ray $L_{1}$, through zero, making an angle $\pi / n$ with the positive real axis. Draw a circle $\mathcal{C}_{1}$ with center 1 and radius

$$
R=\frac{\sin \left(\frac{\pi}{n}\right)}{\cos \left(\frac{\pi}{2 l}\right)} .
$$

This implies that the angle between $L_{1}$ and $\mathcal{C}_{1}$ is exactly $\pi /(2 l)$. Choose $\lambda \in(\rho,(1+R) /(1-R))$, where $\rho>1$ is a solution to the quadratic equation

$$
\rho^{2}\left(1-R^{2}\right)-2 \rho\left(1+R^{2} \cos \left(\frac{\pi}{l}\right)\right)+1-R^{2}=0
$$

Set $\mathcal{C}_{\lambda}$ the circle with center $\lambda$ and radius $\lambda R$. The angle between the positive real axis and $\mathcal{C}_{\lambda}$ is also $\pi /(2 l)$ (this is consequence of the fact that $\mathcal{C}_{\lambda}=A\left(\mathcal{C}_{1}\right)$, where $\left.A(z)=\lambda z\right)$.

The angle between the circles $\mathcal{C}_{1}$ and $\mathcal{C}_{\lambda}$ varies continuously between 0 and $(l-1) \pi / l$ for $\lambda$ varying continuously between in $(\rho,(1+$ $R) /(1-R))$. 
The restriction $1 / l+2 / k<1$ means that for any $k \geq 2$ we can find $\lambda \in(\rho,(1+R) /(1-R))$ such that the angle between the two circles is exactly $\pi /(2 k)$. Moreover, the intersection of the two circles occurs in the interior of the sector

$$
\left\{z \in \mathbb{C}: \frac{-\pi}{n}<\operatorname{Arg}(z)<\frac{\pi}{n}\right\} .
$$

We can find a transformation $x$ in $\operatorname{PSL}(2, \mathbb{C})$ of order $k$ whose fixed points are the two intersection points of both circles and mapping $\mathcal{C}_{1}$ onto $\mathcal{C}_{\lambda}$. The group $G$ generated by $x$ and $r$ (as in the first construction) has presentation

$$
G=\left\langle x, r: x^{k}=r^{n}=\left(r^{-1} x^{-1} r x\right)^{l}=1\right\rangle
$$

Figure 13.

and is a Kleinian group. $\mathbb{H}^{3} / G$ is the hyperbolic orbifold with underlying topological space $S^{3}$ minus two points and branch locus as shown in figure 13 (it has infinite volume since the group $G$ has nonempty region of discontinuity on the the Riemann sphere).

Using the fact that we can permute the roles of $k$ and $n$ in our construction, we have that the only exceptions to this construction is the one we have stated, that is, $1 / l+2 / k<1$. 


\subsection{Summary.}

Both constructions give a faithful representation of the generalized triangle group

$$
G=\left\langle x, r: x^{k}=r^{n}=\left(r^{-1} x^{-1} r x\right)^{l}=1\right\rangle,
$$

as a discrete group in $\operatorname{PSL}(2, \mathbb{C})$, with the exceptions of the following triples $(n, k, l) \in\{(3,2,2),(3,2,3),(3,3,2),(4,2,2)\}$ (this is $[5$, theorem 1]). Three of these cases (that is, with the exception of $(3,3,2)$ ) can be represented inside the isometry group of $S^{2} \times \mathbb{R}$ (see remark of Section 3$)$. The case $(3,3,2)$ is in part discussed in the last part of Section 7.

Our constructions also permit us to say that we obtain finite covolume representations only in the first construction for $n \in\{3,4\}$.

The group $J$ generated by the transformations $x_{1}, \ldots, x_{n}$, where $x_{1}=x$ and $x_{i+1}=r x_{i} r^{-1}$, has presentation

$$
G=\left\langle x_{1}, \ldots, x_{n}: x_{i}^{k}=\left(x_{i+1} x_{i}^{-1}\right)^{l}=1\right\rangle,
$$

that is, it is a generalized Coxeter group. Since a generalized Coxeter group

$$
G=\left\langle x_{1}, x_{2}: x_{i}^{k}=\left(x_{i+1} x_{i}^{-1}\right)^{l}=1\right\rangle,
$$

is in fact a triangle group, we have the following

Theorem 3. For each triple of integers $(n, k, l)$ with $n \geq 2, l \geq 2$ and $k \geq 2$ such that $(n, k, l)$ is different from $(3,3,2)$, there is a discrete and faithful representation of the generalized Coxeter group

$$
G=\left\langle x_{1}, \ldots, x_{n}: x_{i}^{k}=\left(x_{i+1} x_{i}^{-1}\right)^{l}=1\right\rangle,
$$

as group of isometries in one of the geometries $\mathbb{R}^{3}, \mathbb{H}^{2} \times \mathbb{R}, S^{2} \times \mathbb{R}, \mathbb{H}^{3}$. Moreover, the geometry is $\mathbb{H}^{3}$ if and only if $(n, k, l)$ is different from $(3,2,2),(3,2,3),(3,3,2)$ and $(4,2,2)$.

\section{References.}

[1] Apanasov, B., Discrete Groups in Space and Uniformization Problems. Mathematics and Its Applications. Kluwer Academic Publishers, 1991. 
[2] Coxeter, H. S. M., Moser, W. O. J., Generators and Relations for discrete Groups. Springer-Verlag, 1980.

[3] Dunbar, W. D., Geometric orbifolds. Revista Mat. Universidad Complutense de Madrid. 1 (1988), 67-99.

[4] Hagelberg, M., Generalized triangle groups and 3-dimensional orbifolds. To appear in Abh. Math. Sem. Univ. Hamburg.

[5] Hagelberg, M., Maclaughlan, C., Rosenberger, G., On discrete generalized triangle groups. To appear in Proc. Edinburgh Math. Soc.

[6] Hagelberg, M., Vesnin, A. Y., On the geometric structure of Thetagraphs. Preprint, 1994.

[7] Helling, H., Mennicke, J., Vinberg, E. B., On some general triangle groups and 3-dimensional orbifolds. Preprint, 1991.

[8] Haeflieger, A., Quach, N. D., Une présentation du groupe fondamental d'une orbifold. Asterisque 116 (1984), 98-107.

[9] Maskit, B., Kleinian Groups. Grundlehren der Mathematischen Wissenschaften 287. Springer-Verlag, 1988.

[10] Scott, W., The geometries of three manifolds. Bull. London Math. Soc. 15 (1983), 407-487.

[11] Thurston, W. P., The geometry and topology of 3-manifolds. Lecture Notes, Princeton Univ., 1980.

[12] Tsaranov, S. V., On a generalization of Coxeter groups. Alg. Groups Geom. 6 (1989), 281-318.

[13] Tsaranov, S. V., Finite generalize Coxeter groups. Alg. Groups Geom. 6 (1989), 421-452.

Recibido: 5 de junio de 1.996

Revisado: 6 de enero de 1.997

Marcel Hagelberg

Université Paul Sabatier

Toulouse, FRANCE

hagelb@cict.fr
Rubén A. Hidalgo*

U.T.F.S.M.

Valparaiso, CHILE

rhidalgo@mat .utfsm.cl

* The second author was supported by Projects FONDECYT 8970007 and UTFSM 971223 